\title{
Comparative Study of Skin Color based Segmentation Techniques
}

\author{
Noor A. Ibraheem \\ Computer Science Deptt. \\ Faculty of Science, AMU, \\ Aligarh, India \\ Computer Science Deptt, \\ College of Science for Women \\ Baghdad University, Iraq
}

\author{
Rafiqul Z. Khan \\ Computer Science Deptt. \\ Faculty of Science, AMU, \\ Aligarh, India
}

\author{
Mokhtar M. Hasan \\ Computer Science Deptt. \\ College of Science for Women \\ Baghdad University, Iraq
}

\begin{abstract}
Segmentation is the classification of the input colored image into skin and non-skin pixels based on skin color information. A wide range of applications that require the segmentation process as a preprocessing operation such as computer vision, face/ hand detection and recognition, medical image analysis, and pattern recognition. Color information is one of the simple cues used for detecting skin color, and the use of proper color space to represent color information of an image is a crucial decision. In this literature different segmentation techniques are presented, examples and comparison between the main three based segmentation techniques are given as well. Skin color modeling based statistical model is explained in detail, with discussion the combination with different segmentation techniques. The selection of appropriate segmentation method depends on the application and system environments. The performance of any segmentation algorithm is quantified using some benchmarking such as recall and precision coefficients, or by calculating the percentage of correct and false detection rates according to the complexion of the technique used.
\end{abstract}

\section{General Terms}

Segmentation Algorithms, Pattern Classification, Clustering.

\section{Keywords}

Color Image Segmentation, Color Space, Pixel Based Segmentation, Edge Based Segmentation, Region Based Segmentation, Gaussian Mixture Model.

\section{INTRODUCTION}

Image segmentation is the process of partitioning an image into understandable non overlapping regions [1]. The purpose of the segmentation is to separate/divide/partition the image into significant meaningful regions representation which simplifies and easy the analysis of the image [2] with considering to a specific image application [1]. The resulted segmented regions should achieve two main characteristics those are similarity (homogeneity) and connectivity [3] and adjacent regions have different properties [2], the homogeneity property satisfied when the pixels within same region share attributes such as; color, intensity, size, shape [3][4], and textural information [3][4], and the region assumed to be connected if for any two adjacent pixels there is a connected path [3][5] exists within the whole region [3][5]. The threshold value can be determined by the resulted connectivity degree of any two adjacent pixels [6]. There two types of connectivity in a region; 4-connectivity, if the laterally for adjacent pixels are only considered to connect [5], and 8-connectvity if the diagonal adjacent pixels are connected [7][5].

The skin segmentation for color images is a crucial step for variety of applications [8] such as machine vision [6], human computer interaction, pattern recognition, image compression [9], traffic control system [6], video surveillance, etc. [8]. Hand segmentation is one of the basis key technologies [10] for gesture recognition and based on complexion segmentation accordingly [10]. One of the commonly helpful cues for hands and faces segmenting are skin color [11][12] and background subtraction [13], since human skin is consistent and different from other objects [12] which produce good results under well constrained environments [14][11]. The problems of hand skin segmentation can be counted in some factors which are; variant illumination, low video quality [13], and intricate background [13].

For segmented the tracking hand two aspects are required; shape and motion [15] motion and shape modeling used with noisy environments [16]. Two different methods for tracking as mentioned in [15] in each frame the hand pose processed separately, and there is no need for any tracking information [15], and the second method use the tracking information [15]. Some tools such as particle filter [16], Kalman filter, and HMM can be used in such methods, however this method required initialization hand location for starting the tracking [15][16]. The low level segmentation problem [16] is referred by engineering [16] the setup of the processed image and in order to simplify the segmentation process; colored gloves, and restricted environment can be imposed to achieve that purpose [16]. Vision based methods depend on some characteristics like skin color and motion, still these methods affected by variance of illumination factor [16].

However, perfect segmentation is hardly to achieve since the overlapping of skin colors with the complex background [10] will continue to the subsequent steps in gesture recognition [16], because of that, the database should be built for simplifying this objective as done in [17] whose built the first database for skin color (the Compaq database), furthermore, other review studies about segmentation can be found in [4][18][19] [20].

This paper is organized as follows. Section 2 provides a summary of color space representations section 3 introduces 
Skin color based model. Section 3 presents segmentation techniques. Section 4 presents a classification of segmentation methods, Section 5 explained Gaussian model classifier. Benchmarking of segmentation performance evaluation is given in Section 6, and other segmentation techniques are explained Section 7, and Finally conclusions are concluded in Section 8 .

\section{COLOR SPACE REPRESENTATIONS}

For colored image processing; several color spaces are commonly applied [18]. Any image acquired by a digital camera(s) or video is decoded by RGB color model [20] which is the most common color space, where the components of each color pixel are defined by red, green, and blue [18] in an orthogonal Cartesian space [18] according to tristimulus theory [20] where the three bands form the human visual system [18]. Color models that depend on human color perception are various [20]. Many color models based on human color, HSV color model one of these models, Munsell has been used by most of them [20].

Numerous color spaces with different characteristics are used for skin color segmentation [21][12], and most of them share same properties [21].

The main four commonly used are RGB, HSV (and similar HSI and HSL), YCbCr (with similar YIQ and YUV, YES), and CIE Lab (and CIE Luv) [22][21]. RGB color space defines the primary colors in three channels, red, green, and blue [12]. The high correlation between these channels [12] leads to a luminance sensitivity color space, and provides RGB color space with different properties for any lighting condition changes [12]. For this reason other color spaces have been used for skin detection purposes [12]. HSI is an expensive transformation [23][12] particularly for images with high resolution [12], however it can be stored beforehand in a look-up table [12][23]. This provides easily retrieving for HSI values [12] and increase processing velocity [23]. In some cases the channel that affected with the illumination is discarded [12], such color spaces named normalized color models such as the normalized-rg, and HS color models which use only the chrominance components of the color model and neglect the illumination component, to reduce the light sensitivity [22]. This simplified color spaces are easily calculated, more compact in representation [12], robust to lighting change conditions [12], and the invariant at uniform scaling of RGB [20].

However a recent study [21] analyzed the affected of skin segmentation techniques on different color model by investigating two characteristics; the choice of proper color space and the use of chrominance components [21] used four popular representative color spaces RGB, HSV, YCbCr, and CIE Lab. Converting RGB to HSV and CIE Lab needs nonlinear transformation while between $\mathrm{RGB}$ to $\mathrm{YCbCr}$ is linear [21] transformation, and infers that the color model used in classification of skin color depends on image format employed and successive steps for image processing applied [21]. For more details about color space transformations refer to [24].

\section{SKIN COLOR BASED MODEL}

Many approaches where proposed for region based segmentation on skin color modeling [12]. Segmentation based skin color are preferred since it is invariant to rotation [25], scaling [25], besides its simplicity and ease to implement [25]. The selection of efficient skin color modeling depends on particular application and the effective color model proper for that application [12]. Some scientists focus on statistical methods such as Gaussian mixture model (GMM) which is a statistical method [17] base on parametric estimation of skin color [26][12][22][21], others used non-parametric statistical modeling [47] such as histogram based-modeling [27][12][17]. In skin color classification process; the pixel is classified as a skin pixel or non-skin pixel based on extracted skin color [21], since the skin color is effected by lighting changes, the selection of a color model that separate illumination components from chrominance components [25] provides an efficient segmentation process.

Some researches use pixel chrominance or normalized color model to produce a system that is robust against lighting perturbations by discarding the brightness value [28][21][29]. Since human skin color might interfere with other skin color in the image or with the complex background; some assumptions are made to the environment or the user [30] for robust segmentation based on skin color [29]. Some researches overcome this problem by using special gloves such as instrumented gloves, color gloves or magnetic markers [11][7][30] to directly measure hand position [31], shape/pose [11][32], location [11][31], and joint angles [32][31]. These devices hitherto obstacle the naturalness and ease of the system. Some researches avoided these difficulties and used range images [30] or depth information that acquired using special kind of camera like Time-of-Flight ToF camera [12]. Object's depth data can be captured by this ToF camera in the prospect for each point (pixel) at high frame rate [6][12] and uses infrared light to measure the distance of the object [12] which is provided with illumination invariant [12] and color invariant [12] properties. Range 5 to $15 \mathrm{~mm}$ for depth resolution besides range 0.5 to 3 meters for distance [12]. The 3D Time-of-Flight (ToF) sensors became more popular recently in human computer interaction (HCI) field since it supplied a gray level image with range information about each pixel more preferable than ToF camera [30].

\section{IMAGE SEGMENTATION}

The goal of image processing operations is to produce better classification of regions of interest by spotting proper features that can be recognize from other image regions and background [43]. For any image segmentation process two characteristics are applied for guiding the classification procedure [15][68]:

1- Discontinuity: Searches for sharp change in the image intensity to detect the edges of a specific object.

2- Similarity: Searches for similar values (color values) between neighbor pixels to join specific image regions.

The resultant of performing segmentation operation is a partitioned image into regions, the similarity measure form each region and the discontinuity measure separate between them [43].

\subsection{Formal Description}

The formal definition of the segmentation problem can be demonstrated in the following form [18][58][45]. The image I is partitioned into a set of $\mathrm{m}$ regions $R_{i}, \mathrm{i}=1 \ldots \mathrm{m}$, which defined as a set of homogenous pixels of the region $R_{i}$, and a logical predicate $\mathrm{P}(\mathrm{R})$ defined for all $\mathrm{R}$ pixels, which must be true for all $\mathrm{R}$ pixels and false for other regions pixels.

- $\quad R_{i}$ is a connected set, $i=1, \ldots, m$ 
- Complete: Image $=\cup R_{i}, i=1, \ldots, m$

- Disjoint subsets: $R_{i} \cap R_{j}=\emptyset, \forall i \neq j$

- Uniform regions: $P\left(R_{i}\right)=$ True,$\forall i$

- Maximal regions: $P\left(R_{i} \cup R_{j}\right)=$ False, $\forall i \neq j$

\subsection{Classification of Segmentation Methods}

Segmentation methods classified into four kinds, and the selection of the proper method depends on the specific application and working environments. These methods are: pixel, edge, region, and model based segmentation. In the following subsections, types of segmentations based techniques are briefly introduced.

\subsubsection{Pixel Based Segmentation}

Point based or pixel based segmentation, also known as thresholding [44] is the simplest approach for segmenting images [43], depending on gray-level values to segment image pixels [45]. For skin color classification several algorithms have been suggested [21], which include Bayesian classifier [2][46], piecewise linear classifiers [8], histogram based thresholding [44][18][20], histogram [27], fuzzy clustering [20],Gaussian classifiers [14][10][40][39][12][22] [41], Neural Networks (NNs) [36][18][47][48][49], Genetic algorithms (GA) [50], and Hidden Markov Model (HMM) [16].

Table 1: Input device, background type, and domain application of some discussed methods.

\begin{tabular}{|c|c|c|c|}
\hline Reference & Input device & Type of background & Application domain \\
\hline$[7]$ & Webcam/ data glove & cluttered & Gesture recognition \\
\hline [14] & PC camera & Complex & Face recognition \\
\hline [16] & Video/ colored glove & complex & Gesture recognition \\
\hline [21] & $\begin{array}{l}\text { Digital camera/web } \\
\text { images }\end{array}$ & Complex & $\begin{array}{l}\text { Skin segmenting using } \\
\text { color pixel classification }\end{array}$ \\
\hline [22] & Single camera & complex & Face detection \\
\hline [25] & Single camera & Plain/ uniform & Hand gesture recognition \\
\hline [29] & $\begin{array}{l}\text { digital camera, with a } \\
\text { sensor }\end{array}$ & Uniform & Hand gesture recognition \\
\hline [30] & $\begin{array}{l}\text { 3D Time-of-Flight ToF } \\
\text { camera }\end{array}$ & Simple / complex & Hand segmentation \\
\hline [31] & Webcam & Uniform & $\begin{array}{l}\text { ASL American sign } \\
\text { language }\end{array}$ \\
\hline [32] & Webcam & - & Analysis gestures features \\
\hline [33] & $\begin{array}{l}\text { Two image capture cards } \\
\text { at a resolution of } \\
320 \times 240\end{array}$ & cluttered & $\begin{array}{l}\text { 3D pointing gesture for } \\
\text { HCI }\end{array}$ \\
\hline [34] & Single camera & Uniform & - \\
\hline [35] & Video camera & Cluttered & $\begin{array}{l}\text { Piano hand and finger } \\
\text { detection }\end{array}$ \\
\hline [36] & $\begin{array}{l}2,3 \text { cameras, and a frame- } \\
\text { grabber }\end{array}$ & - & Gesture recognition \\
\hline [37] & Single camera & Complex & $\begin{array}{l}\text { Janken (Japanese finger } \\
\text { guessing game) }\end{array}$ \\
\hline [38] & $\begin{array}{l}\text { Twin camera and } \\
\text { projection table }\end{array}$ & Uniform & Visual gesture interfaces \\
\hline [39] & video & Uniform & Gesture recognition \\
\hline [40] & $\begin{array}{l}\text { Video bumblebee stereo } \\
\text { camera system }\end{array}$ & complex & $\begin{array}{l}\text { Recognize and isolate } \\
\text { Arabic numbers }(0-9)\end{array}$ \\
\hline [41] & $\begin{array}{l}\text { Web (Michigon face } \\
\text { database) }\end{array}$ & Complex/ black & $\begin{array}{l}\text { Application human skin } \\
\text { color in images }\end{array}$ \\
\hline [42] & Uniform & Simple & $\begin{array}{l}\text { Segmenting simple images } \\
\text { nor real images }\end{array}$ \\
\hline
\end{tabular}




\section{a) Bayesian Classifier with Histogram Technique}

One of the techniques that are used in statistical pattern classification is Bayesian decision rule [21][46]. In this decision rule, the color pixel $\mathrm{X}$ is considered as a skin pixel according to the following equation:

$$
\frac{P(X / \text { skin })}{P(X / \text { nonskin })} \geq T
$$

Where $\mathrm{P}(\mathrm{X} / \mathrm{skin})$ represents the pdf of skin colors, $\mathrm{P}(\mathrm{X} /$ nonskin) represents the pdf non-skin colors and $\mathrm{T}$ represents the threshold value. According to the probabilities of skin and non-skin, and classification costs, the value of $\mathrm{T}$ is computed; however the value of $\mathrm{T}$ is finalized empirically [21]. [2] applied Bayesian rule to assign a probability to decide the belonging elements in the clustered image. [17] applied histogram technique with Bayesian classifier for skin detection process.

\section{b) Piecewise Linear Classifiers}

Piecewise linear classifiers classified as non-parametric method [51] that divides skin and non-skin colors using a piecewise linear decision boundary [28]. It is also known as "explicit skin cluster" classifier which defines clearly the boundaries of clusters in properly selected color space [8]. [8] Suggested a set of fixed thresholds in six color spaces $\mathrm{YCbCr}$, RGB, HSV1, HSV2, HSI, and rgb, using piecewise linear classifier and use genetic algorithm to decide the boundaries of these six classifiers [8].

\section{c) Gaussian Classifiers}

Gaussian classifier has been used for modeling pixels distribution in colored images [12], the conditional-pdf of different class of the skin color feature vector is approximated using parametric form [52][21], which can be modeled as a combination of pdf s multivariate Gaussian, the general form [52][21]:

$$
P(x)=\frac{1}{(2 \pi)^{\frac{d}{2}}|\Sigma|^{2}} e^{\left[\frac{-1}{2}(x-\mu)^{T} \Sigma^{-1}(x-\mu)\right]}
$$

Where $\mathrm{x}$ represents the d-components of the feature vector, $\mu$ represents the mean of each feature, $\sum$ represents the covariance matrix d-by-d, and $|\Sigma|$ represents the determinant. Its main property is the ability to capture and model the data on the dimensions of the feature vector [52]. Fig 1 shows the 1D and 2D Gaussian model. For mixture of K Gaussians the probability is defined as:

$$
\mathrm{P}(\mathrm{x})=\sum_{\mathrm{j}=1}^{\mathrm{K}} \mathrm{w}_{\mathrm{j}} \cdot \mathrm{N}\left(\mathrm{x} \mid \mu_{\mathrm{j}}, \sum_{\mathrm{j}}\right)
$$

Where $w_{j}$ is the weight of the jth Gaussian model.

$$
\sum_{j=1}^{K} w_{j}=1 \text { and } 0 \leq w_{j} \leq 1
$$

$d=1$ :

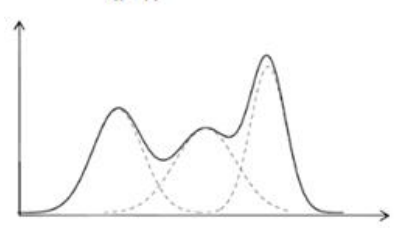

A

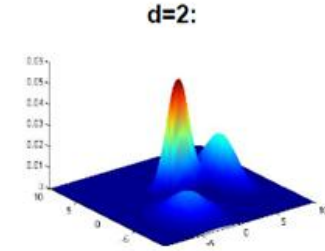

B
Fig 1: Data representation in Gaussian model in 1D and 2D respectively [53].
A lot of authors proposed the use of Gaussian model for image segmenting, either using unimodal Gaussian (GM) [22] or a mixture of Gaussians (MoG) or GMM [12][22]. Expectation maximization EM algorithm [54][26][28] usually used to estimate Gaussian parameters, and K-mean clustering algorithm for parameters initialization [12]. [26] applied two bidimensional Gaussians with parameters (Mean, variance) and EM for parameters estimating, for segmenting images using chrominance components of $\mathrm{YPbPr}$ color space.

\section{d) Neural Network}

For any artificial system application, some aspects should be considered; 1) System robustness against noise and imperfection environments, 2) Simulate the human information, 3) and finally real time output application system [55]. NNs tried to achieve these aims [55]. Neural networks have a huge connected processors working in parallel [55][56]. Several researches [47][48][49] have proposed the use of NNs in segmenting image [55]. self-organizing map (SOM) and multilayer perceptron (MLP) which is feedforward neural network (classified as nonlinear model [51]) have been used widely in classification, compression, and regression [46][21][55]. [48] classified the image using selforganizing map (SOM) using HSL color space with considering only chrominance components hue and saturation, and ignoring the luminance component. [49] applied SOM algorithm on medical images, empirically discovered that increasing network neuron numbers will improve the performance of segmentation process [49].

\subsubsection{Edge-Based Segmentation}

Edge-base or boundary-based segmentation methods [57] commonly refer to segmenting an image based on the edges among regions [18][9], by searching for edge pixels and connect them to form image contours [45]. However for applying such methods two approaches are founded [44]; manually, by using the mouse to draw lines that represent image boundaries among regions, and automatically, by implementing some edge detection filters [44][9], where the pixels classified into edge or non-edge according to the filter output result [44]. Examples of some edge detection filters are: canny edge detector [57], Prewitt's filter [44], Laplacian of Gaussian filter [44], global processing edge based method (Hough Transform) [45], Sobel edge detector [26], watershed segmentation algorithm [9].

\section{a) Canny Edge Detector}

Canny edge detector defined as the standard benchmark for comparing with other edge detection methods [57], canny detector algorithm consists of four steps as mentioned in [57]: (1) uses Gaussian convolution to blur the image and reduce noise effects, (2) uses sobel operator and find the 2D spatial gradient to determine the edge directions and strength, (3) uses edge direction to search along the edges and suppress any non-edge pixels, and (4) eliminates the broken edges using two high and low thresholds [57], pixels above the high threshold are edge pixels and between the high and low thresholds which are adjacent to the edge is considered as edge pixels also. However canny can find pixels near the edge, but less in finding the exact edges [57].

\section{b) Laplacian of Gaussian Filter}

The Laplacian filter is a derivative filter applied to find the regions of rapid intensity change [68][67]in an image to detect the edges [68][69]. Since Laplacian filter is a derivative filter, it is usually applied to images that have smoothed using some filters (such as a Gaussian smoothing filter) to minimize the 
noise sensitivity [68][69]. The filters procedure normally carried out on a gray level image and produce new edged gray level image [69].

\section{c) Watershed Segmentation Algorithm}

Watershed segmentation algorithm considered as a hybrid method between edge-based and region-based segmentation [44]. The aim of watershed segmentation algorithm is to discover the "watershed lines" [9] in the input image to segment the prominent regions [9]. The basic idea of the watershed algorithm can be explained as flooding water process in the topographic surface, streaming form the lowest basin to the highest peak [9][20], the water move through holes and flood the surface [45]. To prevent merging of two different basins a dam is constructed, so that finally the boundaries of connected dam considered as the watershed edges [45]. The main advantage of this method is the continuity of region's boundaries, however the resultant segmented image suffer from over-segmented regions and it is time-consuming as well [9].

\subsubsection{Region-Based Segmentation}

Region-based segmentation partitions an image into regions or groups of similar pixel depending on some properties [58]. Its principle depends on the idea that neighboring pixels within the same region have same value [9]. This idea can be implemented by comparing each pixel with its neighbors in a particular region [9], and according to the crucial similarity condition the pixel is decided to belong to a specific region [9]. In the segmentation process feature image is used instead of original input image, the feature image is represented with small neighborhoods which forms a regions [43]. Regionbased segmentation technique required the use of proper thresholding methods [57], and the noise has great impact on the output result [9]. Some skin classification methods based region based are: region growing, region splitting, region merging [20], split and merge [20], NNs [18].

\section{a) Region growing Techniques}

In these methods, uniform or homogenous regions (seeds) of an image are obtained using growth techniques [20][18], to join the surrounding neighborhoods together and recognize this region from other regions [20]. The idea behind the growth process begins from a preselected pixel (seed) [18], gradually agglomerates pixels that achieve homogeneity characteristic between the joined pixels, this operation stops when number of points that can be added to the region is zero[18].

\section{b) Split-and-merge Techniques}

These methods are the inverse to region growing techniques in which it starts from inhomogeneous image classification and continue splits the image into subdivided regions until homogeneous regions are obtained by applying some data structure algorithms [18]. The merging process joined the neighboring regions to achieve homogeneity demands [18] on a uniform image region [18][ 20].

\subsubsection{Model-Based Segmentation}

Model-based segmentation methods required more information about the image (more than the local neighborhood information [43]) such as objects geometrical shape [43] or the repetitive form geometry [6] of a specific region of interest [43]. This method demands (1) to register the training sample, (2) to represent the probability of the registered samples, and (3) finally inference between the model and the image statistically [6]. However model-based methods can be implemented if the exact object shape is known in the image [43]. Examples of model based segmentation are: Gaussian model, Decoder-guided systems could be considered as model-based segmentation as well [6]. Fig 2 explains the taxonomy of segmentation techniques, and Fig 3 shows examples of segmentation methods.

\section{PREPROCESSING STEPS}

Image preprocessing steps are required to achieve an efficient segmentation method. Smoothing the image is one of the needed operations to complete the segmenting process and reduce noise affect as well. [37] applied 5x5 pixel edge smoothing filter, then the smoothed image is binarized using Otsu's thresholding method [37]. [35] used median filter for smoothing the image.

Other methods used image restoration techniques [63] under the continuum and discrete derivatives filters to remove image noise which are: isotropic and anisotropic filters. Isotropic filter removes the noise form the image and the Anisotropic filtering filter improves image quality on scene surfaces that have oblique angles [63].

In [31], they applied canny edge detection filter after resizing the image into 80x64, where canny filter achieve better results than sobel edge filter [31], however canny filter produces more details than required, for this problem thresholding technique applied to overcome such cases [31].

Morphological operations are applied in some researches [7][35][14] to remove some errors[40], and get rid of unwanted details [2]. [7] applied opening operation followed by morphological closure process. Also [61] performed open and close morphology operations to remove speckle noise and small false blob, while [35] applied morphological dilation, [14] used morphological dilation and erosion operations to extract skin pixel [14]. [2][40] used morphological operations as a preprocessing methods for errors removing.

\section{GAUSSIAN MODEL CLASSIFIER}

Since the distribution of skin and non-skin pixels is unimodal [51]. GMM provides acceptable results with minimum set of training data [12], requires low storage system [12], and usually used to train skin pixels [12]. When modeling the distribution using GMM, two important aspects are required: parameters estimating and the number of mixture component [51].

\subsection{Initial values for Gaussian}

The initial values for Gaussian classifier parameters are mean, variance and weight [61]. They can be calculated in two ways, either manually or using particular algorithm. K-means algorithm usually used to set the initial parameters of GMM [40][51].

\subsection{Estimating Parameters}

For estimating GMM parameter values usually EX algorithm is used [51][17][40][41], EM algorithm is used to estimate the parameter's maximum likelihood which are mean vector, covariance matrix and mixture weight of image skin pixels [40]. 


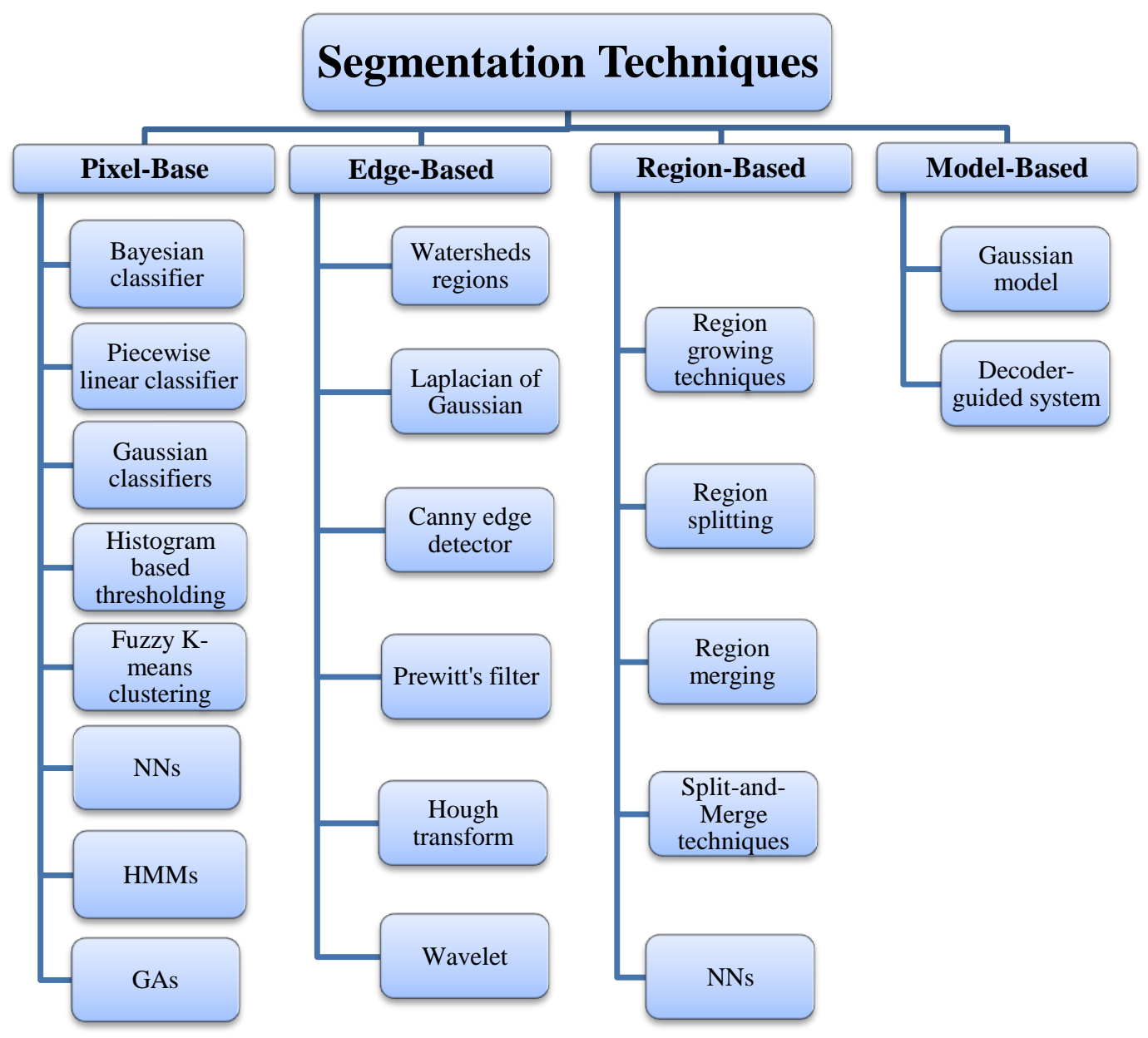

Fig 2: The general segmentation based techniques.
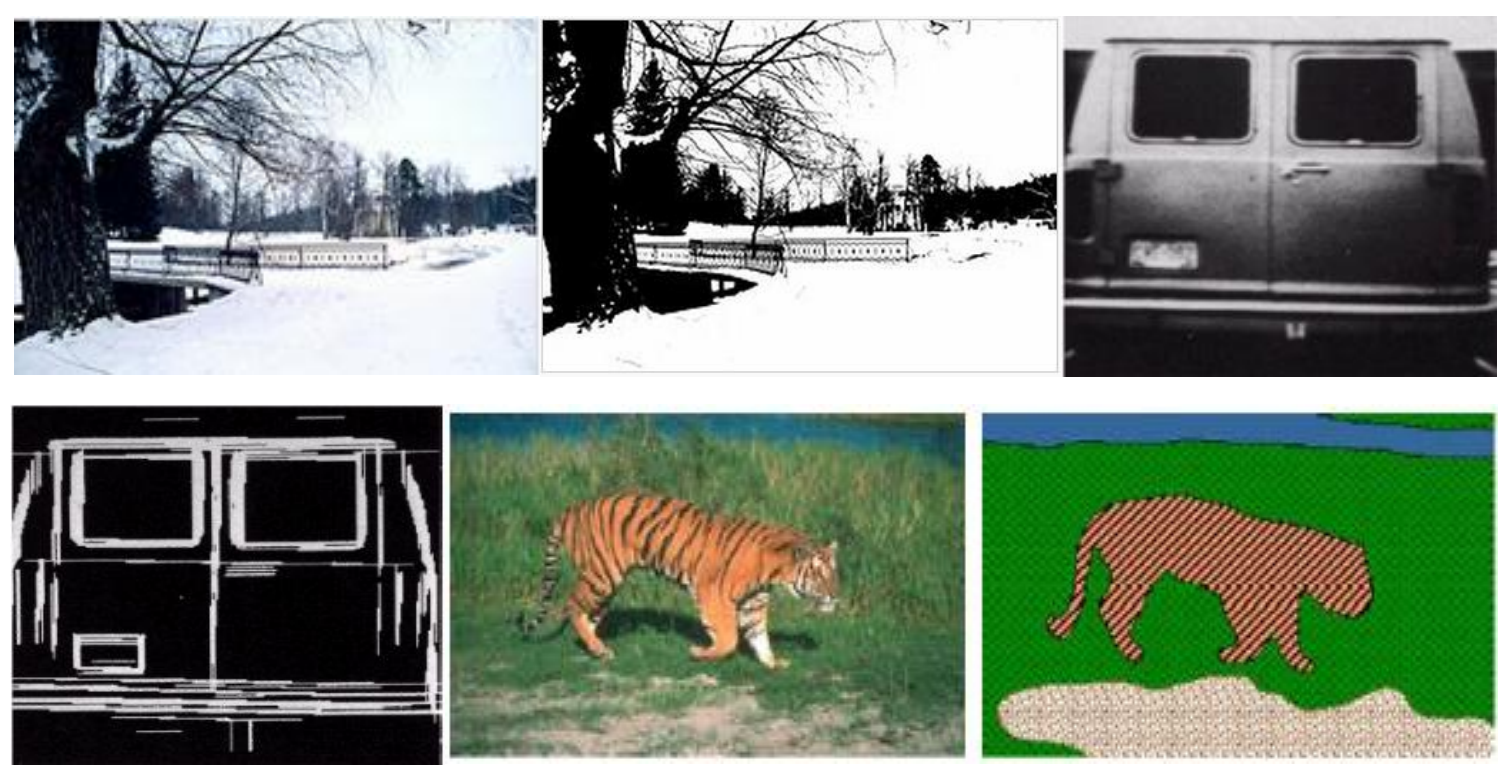

$a b c$

$d$ e $f$

(a), (c), and (e) are the original images, (b) pixel based-thresholding- segmentation result [6], (d) Edge-based segmentation result [60], and (f) region Segmented Image [57].

Fig 3: Segmentation based techniques. 
Table 2: Comparison between the main three based segmentation techniques [59][45].

\begin{tabular}{|c|c|c|c|}
\hline Type & $\begin{array}{l}\text { Pixel-Based } \\
\text { Segmentation }\end{array}$ & Edge-Based Segmentation & Region-Based Segmentation \\
\hline $\begin{array}{l}\text { Method } \\
\text { description }\end{array}$ & $\begin{array}{l}\text { The simplest method for } \\
\text { segmenting image } \\
\text { depending } \\
\text { thresholding value. }\end{array}$ & $\begin{array}{l}\text { Segmenting an input image } \\
\text { using edges (boundaries) } \\
\text { between image regions }\end{array}$ & $\begin{array}{l}\text { Segmenting an input image by } \\
\text { partitioning it into regions (coherent } \\
\text { regions) with similar characteristics } \\
\text { which represent objects }\end{array}$ \\
\hline Computations & $\begin{array}{l}\text { Computations based on } \\
\text { pixel values }\end{array}$ & $\begin{array}{l}\text { Computations for Segmenting } \\
\text { an image based on pixel } \\
\text { differences. }\end{array}$ & $\begin{array}{l}\text { Computations for Segmenting an image } \\
\text { based on pixel similarities. }\end{array}$ \\
\hline Boundaries & - & $\begin{array}{l}\text { Boundaries not necessarily to } \\
\text { be closed. }\end{array}$ & Boundaries should be closed \\
\hline $\begin{array}{l}\text { Multi-spectral } \\
\text { images }\end{array}$ & - & $\begin{array}{l}\text { No enhancement } \\
\text { segmentation is detected for } \\
\text { multi-spectral images. }\end{array}$ & $\begin{array}{l}\text { Multi-spectral images enhance the } \\
\text { segmentation. }\end{array}$ \\
\hline $\begin{array}{l}\text { Disconnected } \\
\text { regions }\end{array}$ & - & $\begin{array}{l}\text { Easily deals with gaps } \\
\text { produced by occlusion. (for } \\
\text { example Hough transform). }\end{array}$ & $\begin{array}{l}\text { Difficult to find objects that extended to } \\
\text { multiple disconnected regions. }\end{array}$ \\
\hline $\begin{array}{l}\text { Region } \\
\text { membership } \\
\text { Decisions }\end{array}$ & - & $\begin{array}{l}\text { Decisions about edges are more } \\
\text { affordable and applicable than } \\
\text { regions based methods. }\end{array}$ & $\begin{array}{l}\text { Decisions about region membership are } \\
\text { often more difficult than applying edge } \\
\text { detectors. }\end{array}$ \\
\hline $\begin{array}{l}\text { Partitioning } \\
\text { regions }\end{array}$ & - & $\begin{array}{l}\text { Threshold dependent. For a } \\
\text { high threshold, the objects will } \\
\text { be overlapped and mixed, and } \\
\text { for a low threshold, over } \\
\text { segmented might arise. }\end{array}$ & $\begin{array}{l}\text { If the image partitioned into over } \\
\text { segmented regions which is smaller than } \\
\text { the actual regions required, or } \\
\text { segmented into wide regions larger than } \\
\text { the required objects, multiple objects } \\
\text { might be crossed and passed over other } \\
\text { objects boundaries. Tradeoff is required }\end{array}$ \\
\hline Robustness & - & $\begin{array}{l}\text { Less robust since there is not } \\
\text { enough information available } \\
\text { for edge detection methods. }\end{array}$ & $\begin{array}{l}\text { Robust than edge methods since regions } \\
\text { contain many pixels in a specific domain } \\
\text { with information characterizes that } \\
\text { region, texture can be used to detect } \\
\text { regions as well. }\end{array}$ \\
\hline Noise images & - & $\begin{array}{l}\text { Inefficient results obtained } \\
\text { from images with noise, smooth } \\
\text { transitions, low contrast, and } \\
\text { occlusions. }\end{array}$ & $\begin{array}{l}\text { Easy to find regions in noise images, } \\
\text { where region growing techniques are } \\
\text { good in this field. }\end{array}$ \\
\hline $\begin{array}{l}\text { Combining } \\
\text { methods }\end{array}$ & - & $\begin{array}{l}\text { Better results when combined } \\
\text { with region based for example } \\
\text { watersheds region algorithm }\end{array}$ & $\begin{array}{l}\text { Better results when combined with edge } \\
\text { based for example watersheds region } \\
\text { algorithm }\end{array}$ \\
\hline Techniques & $\begin{array}{l}\text { Bayesian classifiers, piece } \\
\text { wise, Gaussian models, } \\
\text { thresholding, Histogram } \\
\text { thresholding, NNs, GAs, } \\
\text { HMMs. }\end{array}$ & $\begin{array}{l}\text { Canny edge detector, Laplacian } \\
\text { of Gaussian edge filter, Hough } \\
\text { transform, Prewitt's filter }\end{array}$ & $\begin{array}{l}\text { Region growing, Split and merge, region } \\
\text { splitting, region merging, NNs. }\end{array}$ \\
\hline
\end{tabular}




\subsection{Number of Mixture Component}

The number of Gaussian mixture component can be decided in two ways: either empirically by the user or determined using optimization criteria [51]. Empirically, for example, $\mathrm{k}=5$ in [28], $\mathrm{k}=4$ in [40], $\mathrm{k}=2$ in [41] $\mathrm{k}=10$ in [42], while [51] applied Minimum Description Length MDL algorithm to determine the $\mathrm{k}$ component.

\subsection{Methods used Gaussian Classifiers}

Various methods used GM and GMM, [10] Combines two complexion models by using Gaussian distribution in the $\mathrm{YCbCr}$ space and $\mathrm{Nrg}$ space, and a background model to perform robust segmentation method. For finding the complexion sampling, mean value and covariance matrix are produced using Gaussian model maximum likelihood estimation. Using $\mathrm{YCbCr}$ color space only produced an overlapping of skin likely complexion regions [10], so the $\mathrm{Nrg}$ space solved this problem using the following condition:

$$
r \in[0.33,0.51], g \in[0.28,0.35], \text { even. } r>g
$$

Against any environment changes the system can easily updates the background model with the new requirements [10]. If $\mu_{i}(t)$ and $\sigma_{i}^{2}(t)$ are the expected value and the variance of color distribution at time $t$ of point $i$, and $y_{i}(t)$ is the color value at time $t$ of point $i$. Then the $\mu_{i}(t)$ and $\sigma_{i}^{2}(t)$ at time $t+1$ is:

$$
\begin{gathered}
\mu_{i}(t+1)=\left\{\begin{array}{c}
(1-\alpha) \mu_{i}(t)+\alpha y_{i}(t),\left(D_{i}=0\right) \\
\mu_{i}(t) \quad,\left(D_{i}=1\right)
\end{array}\right. \\
\sigma_{i}^{2}(t+1)=\left\{\begin{array}{cc}
(1-\alpha) \sigma_{t}^{2}(t)+\alpha\left(y_{i}(t)-\mu_{i}(t)\right)^{2}, D_{i}=0 \\
\sigma_{t}^{2}(t) \quad, D_{i}=1
\end{array}\right.
\end{gathered}
$$

In [40] they applied skin segmentation using GMM over $\mathrm{YCbCr}$ color space, and modeling the skin pixel using $\mathrm{K}$ means clustering algorithm to initial mean vector and covariance matrix, and used EM algorithm to estimate the maximum likelihood of the parameters (Mean, covariance matrix and mixture weight) [40]. The system trained with 200 video samples and tested with 98 video samples. In [23] combination of GMM and histogram-based methods to locate the hands, where GMM used for offline training system, and the histogram used for online in real system [23] since the former is sensitive to changes in lightings or users, the second method used to overcome these obstacles [23]. For offline training the GMM are used to model the skin distribution using r-g color space and online testing used HS color model. HSI color space registered an efficient performance with histogram classifier, but its transformation is highly computational cost. To solve this problem a look-up table
(LUT) is used to store the beforehand HSI values and increase the system velocity [23]. After the segmenting, some post processing filters are applied such as median filter to remove the noise and filter-based size to discard any unwanted objects except the hand and the face [23]. [12] Segmented the hand object using combination of skin color based model and depth information of the ToF camera [12]. [12] Applied the same system performed in [23] for skin color detection which combine the GMM and the histogram based Bayes model for offline training and online testing system.

[51] applied GMM to estimate the probability density function (pdf) of human skin color. K-means algorithm used to determine the initial parameters configuration and EM algorithm used to estimate the parameters, and the number of parameters decided using Minimum Description Length (MDL) algorithm. The GMM applied on three different color models which are YES, chromatic space and log-opponent, for the YES color model only the $\mathrm{E}$ channel is used to reduce interference between skin and non-skin pixel data [51]. The number of samples used for training was 144 colored images, with 15,345 skin pixels (in 42 images) and 83,353 non-skin pixels (in 102 images), and for testing was 60 different colored images, with 13,345 skin pixels and 28,669 non-skin pixels. [62] used GMM for modeling face color distribution over HS space, and EM algorithm used to learning a GMM by generating maximum likelihood algorithm [62]. However GMM is used for dynamic face tracking as well by estimating the object (face) position and the bounding box size around the object [62], the box used to focus the processing on the selected area [62]. The position and the size of the box are computed by calculating the mean $\mathrm{m}^{\mathrm{t}}=\left(\mathrm{m}_{\mathrm{x}}, \mathrm{m}_{\mathrm{y}}\right)$ and standard deviation $\sigma^{\mathrm{t}}=\left(\sigma_{\mathrm{x}}, \sigma_{\mathrm{y}}\right)$ of the local color probability distribution [62] in the box bounding that is centered $\mathrm{m}^{\mathrm{t}-1}$ at time $\mathrm{t}$ in the image [62], and the dimensions of the bounding box are scaled to determine the dimensions of the searching box at time t-1 [62]. [21] compared the performance of three classifiers: GM and GMM, histogram based Bayesian classifier and Neural Network NN, and discovered that the latter two classifiers have better performance than the former (GM and GMM), with number of samples for training was 2500 images with 116.6 million for skin pixels and 564.1 million for non-skin pixels, and for testing was 1500 images with 92.8 million skin pixels and 337.7 million for non-skin pixels. [42] presented GMM with the modified EM algorithm named EM-MAP (Expectation Maximization-Maximum a Posterior) algorithm. The EM-MAP algorithm is a pixel labeling method that assigns each segmented object with different kinds of labels. The proposed algorithm uses Bayes rule and a sequence of prior's probability followed by sequence of posterior probability until it reach to a convergence. However the proposed EM-MAP algorithm has some problems in deciding the required prior parameters such as: 1) number of classes, 2) weights, 3) means, 4) and variances. Practically these parameters are estimated by representing the histogram of the required image which will provide the initial values of the parameters, however the resultant information have maximum entropy as well [42]. When the EM-MAP algorithm convergence after some iterations, stability in entropy posterior probability is achieved [42]. Although the suggested algorithm above is suitable for simple images, but it is not applicable on real images where the segmented image relies on modeling the features [42]. Table 3 lists some details about Gaussian model used in various segmentation systems. 
Table 3: Methods Applied Gaussian Model.

\begin{tabular}{|c|c|c|c|c|c|}
\hline Reference & $\begin{array}{c}\text { Gaussian } \\
\text { Type }\end{array}$ & Color Model & $\begin{array}{l}\text { Number of } \\
\text { Samples }\end{array}$ & $\begin{array}{c}\text { Initial Configuration } \\
\text { of Gaussian } \\
\text { Parameters }\end{array}$ & $\begin{array}{c}\text { Estimate the } \\
\text { Maximum Likelihood } \\
\text { of Parameters }\end{array}$ \\
\hline [10] & $\begin{array}{l}\text { Gaussian } \\
\text { distribution }\end{array}$ & $\begin{array}{l}\text { YCbCr space. and } \mathrm{Nrg} \\
\text { space with the } \\
\text { background model }\end{array}$ & $\begin{array}{l}20 \text { samples are } \\
\text { identified }\end{array}$ & - & $\begin{array}{l}\text { Maximum Likelihood } \\
\text { Estimation (MLE) }\end{array}$ \\
\hline [40] & GMM & YCbCr space. & $\begin{array}{l}20 \text { samples for } \\
\text { training, and } 10 \text { for } \\
\text { testing }\end{array}$ & $\begin{array}{l}\text { K-means clustering } \\
\text { algorithm }\end{array}$ & EM algorithm \\
\hline [51] & GMM & $\begin{array}{l}\text { Normalized } \mathrm{CbCr}, \mathrm{Yes} \text {, } \\
\text { log-opponent color } \\
\text { space }\end{array}$ & $\begin{array}{l}144 \text { images for } \\
\text { training data, and } 60 \\
\text { images for testing }\end{array}$ & $\begin{array}{l}\text { K-means clustering } \\
\text { algorithm }\end{array}$ & EM algorithm \\
\hline [23] & $\begin{array}{l}\text { GMM and } \\
\text { histogram based } \\
\text { Bayes method }\end{array}$ & HS and rg color spaces & $\begin{array}{l}100 \text { samples for } \\
\text { training, and } \\
400 \text { for validation }\end{array}$ & - & - \\
\hline [12] & $\begin{array}{l}\text { GMM and } \\
\text { histogram based } \\
\text { Bayes method } \\
\text { with depth } \\
\text { information }\end{array}$ & HS and rg color spaces & $\mathrm{N} / \mathrm{A}$ & - & - \\
\hline [62] & GMM & HS space & $\begin{array}{l}\text { Number of training } \\
\text { images per person } \\
\text { from } 21 \text { to } 60 \text {, and } \\
\text { for testing from } 21 \\
\text { to } 53\end{array}$ & 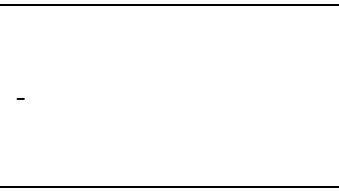 & EM algorithm \\
\hline [28] & GMM & HS space & $\begin{array}{l}163 \text { for training, } 163 \\
\text { test images }\end{array}$ & $\begin{array}{l}\text { Estimated from training } \\
\text { data }\end{array}$ & Restricted EM \\
\hline [42] & GMM & RGB space & Simple images & Histogram based method & EM algorithm \\
\hline
\end{tabular}
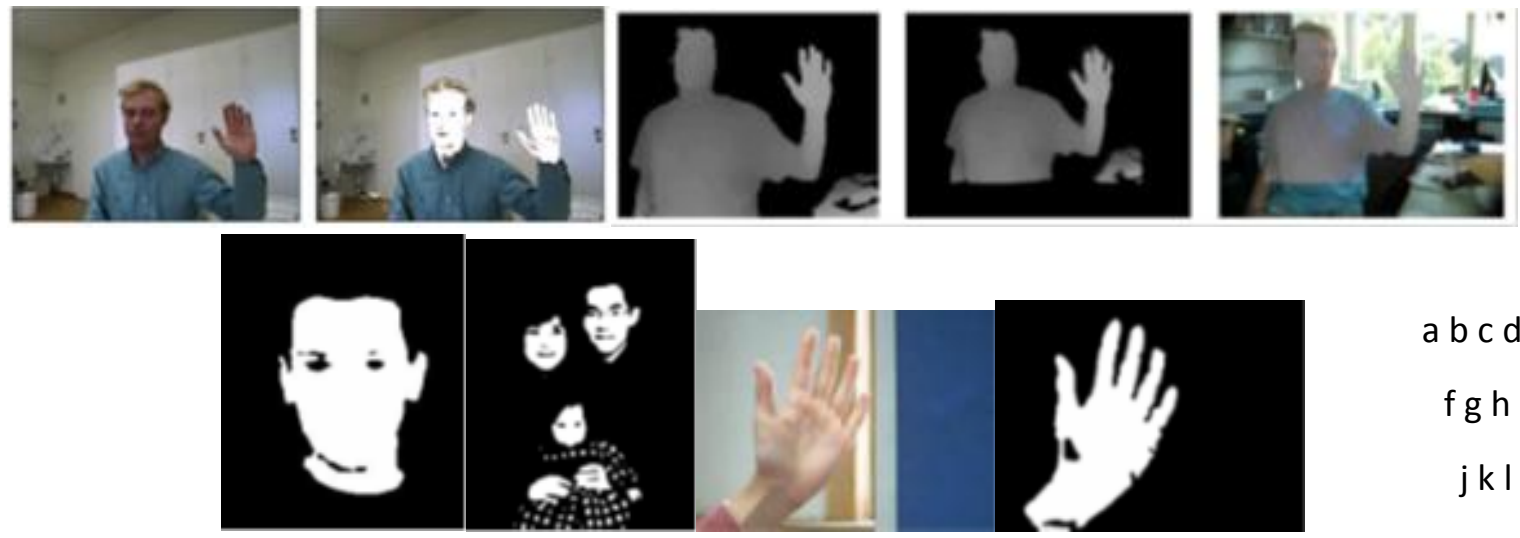

$a b c d e$
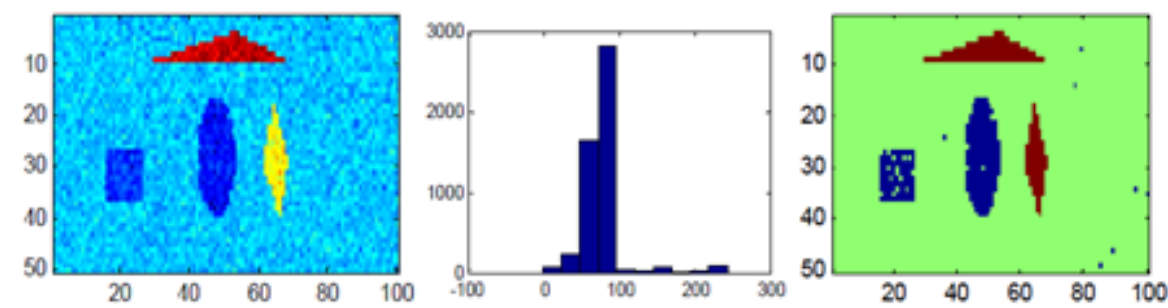

a, b) GMM and Histogram based Bayes methods [23]. c, d, e) GMM and Histogram based Bayes methods combined with depth information

[12]. f, g) GMM model [51]. h, i) GMM with two complexion models YCbCr space and Nrg space, with a background model [10]. j) The input image, k) The histogram of input image, and 1 ) the labeled image after 23 times iteration [42].

Fig 4: Examples of Gaussian Model implementation. 


\section{BENCHMARKING SEGMENTATION PERFORMANCE EVALUATION}

An essential concept in verifying a specified algorithm for any application is the performance evaluation [63]. For evaluating the performance of a particular segmentation technique several benchmarks are proposed by the researchers [6]. Recall and precision measure [8][64][68][65] are used to quantify skin detection method performance [8], and usually used for video segmentation [65].

$$
\begin{gathered}
\text { Recall }=\frac{N_{\text {correct }}}{N_{\text {correct }}+N_{\text {missed }}} \\
\text { Precision }=\frac{N_{\text {correct }}}{N_{\text {correct }}+N_{\text {false }}}
\end{gathered}
$$

Where $\mathrm{N}_{\text {correct }}$ represents the number of skin pixels that correctly detected, $\mathrm{N}_{\text {missed }}$ represents the number of skin pixels that are missed, and $\mathrm{N}_{\text {false }}$ represents the number of skin pixels that falsely detected [65]. Any detection method considered efficient if high recall and precision scores are achieved [8]. In [65] the recall value was $93.2 \%$ and precision value was $91.6 \%$ for video sequence of human detection. [8] adopted a weighted Recall and Precision scores using the fitness function of genetic algorithm to evaluate the boundaries of skin clusters for six skin classifiers, the weights have been selected for acceptable tradeoff between the scores or for either high Recall or high Precision [8].

$$
\text { fitness }=\frac{\text { recall.precision }}{\alpha . \text { recall }+\beta \text {.precision }}
$$

And $\alpha$ and $\beta$ are the weighted coefficients, and assigned the value $\alpha=0.2$ and $\beta=0.8$ for high Recall, $\alpha=0.8$ and $\beta=0.2$ for high Precision, and $\alpha=\beta=0.5$ for tradeoff measure between the two scores [8].

[63] evaluated the performance of topological derivative based segmentation using other performance evaluation benchmarking which is Mean Square Error (MSE) and Peak Signal to Noise Ratio (PSNR) difference and iteration for further evaluation effort, and each input image has different evaluation performance [63]. While [39] applied three metrics for evaluation system performance, Correct Detection Rate ( CDR ), False Detection Rate ( FDR ), and overall Classification Rate ( CR ) for Support Vector Machine (SVM) active learning which are incorporated with region segmentation [39]:

$$
\mathrm{CR}: \frac{N_{S}}{\max \left(N_{S}^{A}, N_{S}^{G}\right)} \times 100 \%
$$

Where $\mathrm{N}_{\mathrm{S}}$ represents the number of skin pixels that are detected using the suggested algorithm and ground truth (the ground truth samples usually segmented manually), $\mathrm{N}_{\mathrm{S}}^{\mathrm{A}}$ represents the number of skin pixels that are detected using the suggested algorithm, $\mathrm{N}_{\mathrm{S}}^{\mathrm{G}}$ represents the number of skin pixels that are detected using the ground truth [39].

The term ground truth stands for information and facts that are collected from real location at the same time when testing the system information [6], it is commonly considered more accurate than the tested proposed system [6]. In [39] the ground truth constructed by randomly chose 240 frames from eight video sequences, and two students manually segmented skin pixels [39].

The proposed model achieves $86.34 \%$ for CDR, 0.96 for FDR, and 76.77 for CR. Other researches used experimental results and comparisons [12] to evaluate the performance of their suggested skin detection methods [33][29][25][27][30].

\section{OTHER SEGMENTATION TECHNIQUES}

Besides the above mentioned approaches, various methods have been applied for segmenting colored images using different segmentation methods [16][39][30][14]. Some researches used color based analysis to detect skin color pixels by transforming to other color spaces, to ease the segmentation problem and overcome some environments affects [29][25][38]. In [29] normalized r-g color model applied to detect skin color based on chrominance values, to reduce the effectiveness of light intensity variations. Figure 5 $(a, b)$ shows a portray version of the aforesaid method. [25] used $\mathrm{YCbCr}$ color space for segmenting hand gestures, figure 5(c, d) shows the application of this method. [38] applied normalized YUV color space followed by some image processing filters; the small holes were filled as well.

Other researches used different methods and algorithms to achieve better skin segmentation results. [34] presented an integrated system that segments and tracks face and hands in a sign language recognition system. Three features are combined for skin color detection: color, motion and position, which represent the information of the foreground pixel, and the predicated moving object position. Each skin object (face and hands) are allocated to a bounding box to ease the discovering of overlapped or occluded objects [34]. Occlusion problem manipulated using Kalman filter, figure $5(\mathrm{~g}, \mathrm{~h}, \mathrm{i}, \mathrm{j})$ shows the segmented hand after some preprocessing operations (converted the image into gray scale, resized the image into $32 \times 32$ scale image, and normalized using color histogram equalization). [63] segmented the hand image using discrete and continuum topological derivatives. For efficient rate of hand detection and noise reduction, two restoration filters are applied; isotropic and anisotropic filters. the restoration filters usually carried out before topological derivatives approach [63]. [36] addressed the problem of segmenting hand image by two steps: firstly hand gesture contour tracking in the space domain, and secondly continuous tracking in time domain [36]. For easily modeling the hand, [36] supposed the hand is free of knuckles and represented the palm with a square and the fingers with rectangular [36] as shown in figure $5(\mathrm{e}, \mathrm{f})$, then matched the model using a lot of template matching samples to overcome translation, scaling, and rotation problems, and used threelayer fully connected feed forward back propagation neural network to adjust the weights. Then active contour was applied for tracking object shapes such as lines, arcs, corners and shadows [36].

Motion-based segmentation methods take into account the necessary features required for gesture motion [16]. [16] proposed segmenting hand gesture using an intermediate grouping module to overcome low-level segmentation errors [16], where the hand image segmented into a set of [16], and use greedy search technique to gather the fragmented regions into acceptable hypotheses based on the size. The generated groups joined with adjacent frames [16] based on some properties like shape, size, and location similarity [16], ultimately matched these HMM gestures with the joined 
groups structure to discover the best grouping for each frame [16]. With this applied method, the color information feature is not required to segment the hand [16].

[39] suggested a generic skin color model for segmentation process based on region information instead of pixel information to lowering the noise appearance and illumination changes [39] and SVM active learning. The system comprises two stages: training stages and segmentation stage. In training stage the generic model performed on a lot of video frames, and applied JESG algorithm [66]to partition each frame into regions, and the training samples consist of skin and non-skin pixels. In segmentation phase the SVM classifier used the training set to segment the testing frames [39]. Figure $5(k, 1)$ shows the results of applying this method. Methods based shape models in segmenting images required adequate difference between object of interest and the background [27]. Some researches detected hand in grey image using Adaboost algorithm [27], same algorithm used for face detection [27]. [27] adopted Adaboost method with some extension by adding new features set as shown in figure 6 (a), the new feature shapes such as sizes and positions are less constrained [27], and allow for overlapping and random areas [27], and for tracking, multimodal technique is used: the optical flow and color cues to achieve stable hand tracking [27].

[37] extracted two images from the input image: skin color image and intensity image, where the RGB input image first converted into HVC (Hue, Value, and Chroma ( or Saturation) ) ( h, v, c ) in Munsell color space [37]. The skin color image is acquired from the table of skin color distribution on the HVC color space [37] that is learned from the sample regions in learning phase, while the intensity image is attained from the RGB color using the following intensity function [37]: $\mathrm{Y}=0.30 \mathrm{r}+0.59 \mathrm{~g}+0.11 \mathrm{~b}$. These two images are multiplied and binarized using Otsu's method to extract the maximum region that represents the hand objects [37]. To achieve robust segmentation, some methods used the range information which can be acquired by various kinds of cameras such as stereo camera [40][30], laser range camera, and TOF camera [12][30]. [30] used the fusion of intensity image and range image generated by $3 \mathrm{D}(\mathrm{ToF})$ camera, as the input image for segmentation process [30], using K-Means Expectation Maximization (KEM) algorithm which proposed to combine the two unsupervised clustering methods the $\mathrm{K}$ means and EM. K-means algorithm applied on the initial clustering to minimize the initial points sensitivity [30] and discover the center of the initial clusters [30], which will used as the initial parameters for EM algorithm which will used to find maximum likelihood estimation [30]. [14] used normalized $\mathrm{r}$-g color model to diminish the effect of lighting changes [14], afterwards used two quadratic polynomials to approximate the upper and lower boundaries of the compact region [14] in the r-g plane distribution. Some modification was applied on the algorithm (by adding additional rules) to enhance the segmentation method [14], Figure 7 shows the distribution of skin pixels over r-g plane. Finally [32] segmented the object of interest manually.
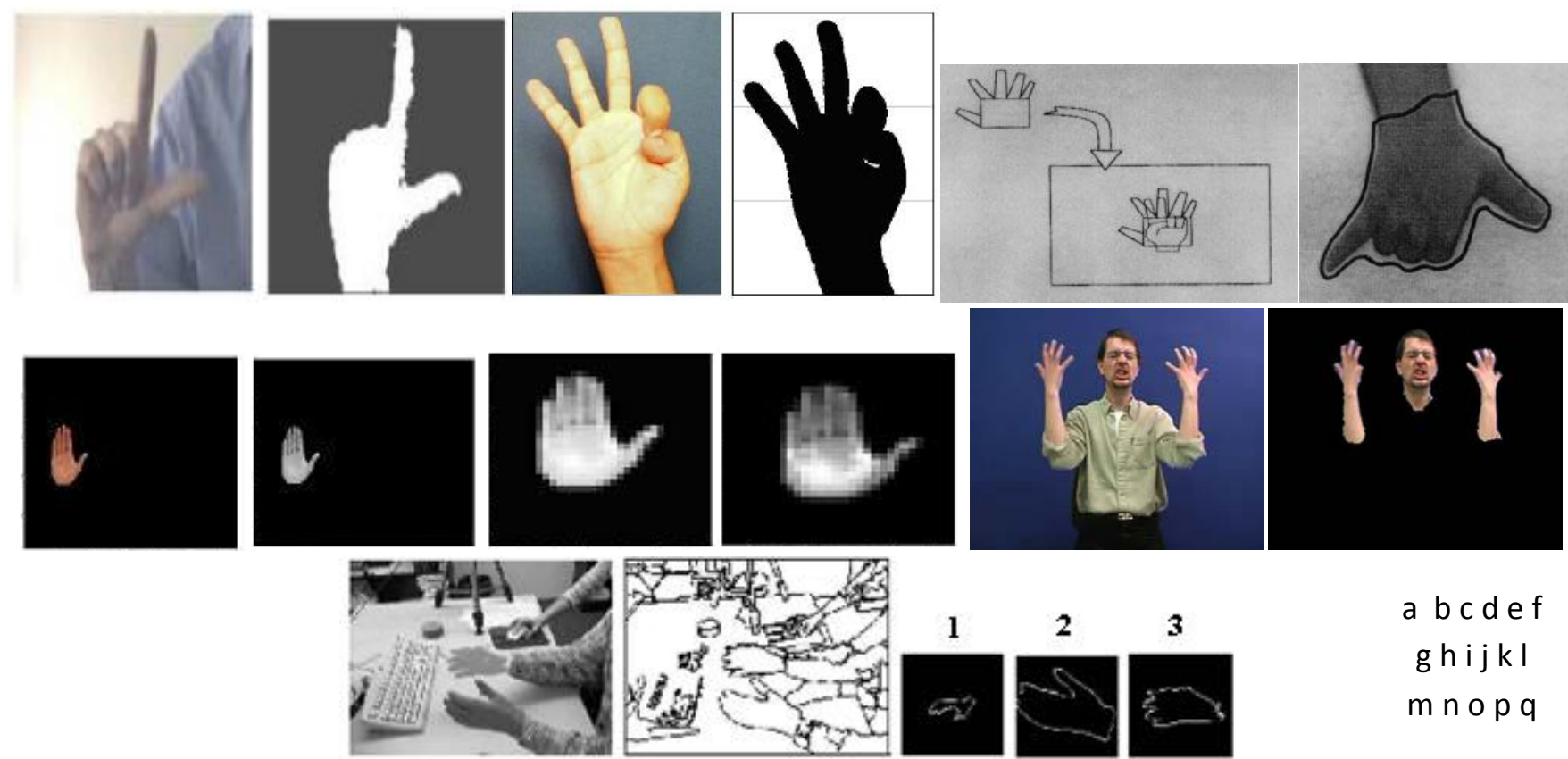

a bcdef

ghijkl

$\mathrm{m} n \circ \mathrm{pq}$

a, b), and c, d) Used color spaces normalized r-g [29], and YCbCr [25] respectively, for segmentation process.

e, f) Contour matching model and tracking [36]. g, h i, j) Used color, motion, and position information, with some preprocessing operations [34]. $\mathrm{k}, 1$ ) applied region information based segmentation and SVM active learning [39]. m) Nominated groups of regions from a specific frame. N) the input image is segmented into boundaries. o) The nominated group for the third hand of $\mathrm{m}$. p) the nominate group for the first hand of $\mathrm{m} . \mathrm{q}$ ) the nominate group for the middle (second) hand of $\mathrm{m}[16]$.

Fig 5: Results of modeling and segmentation some discussed methods. 

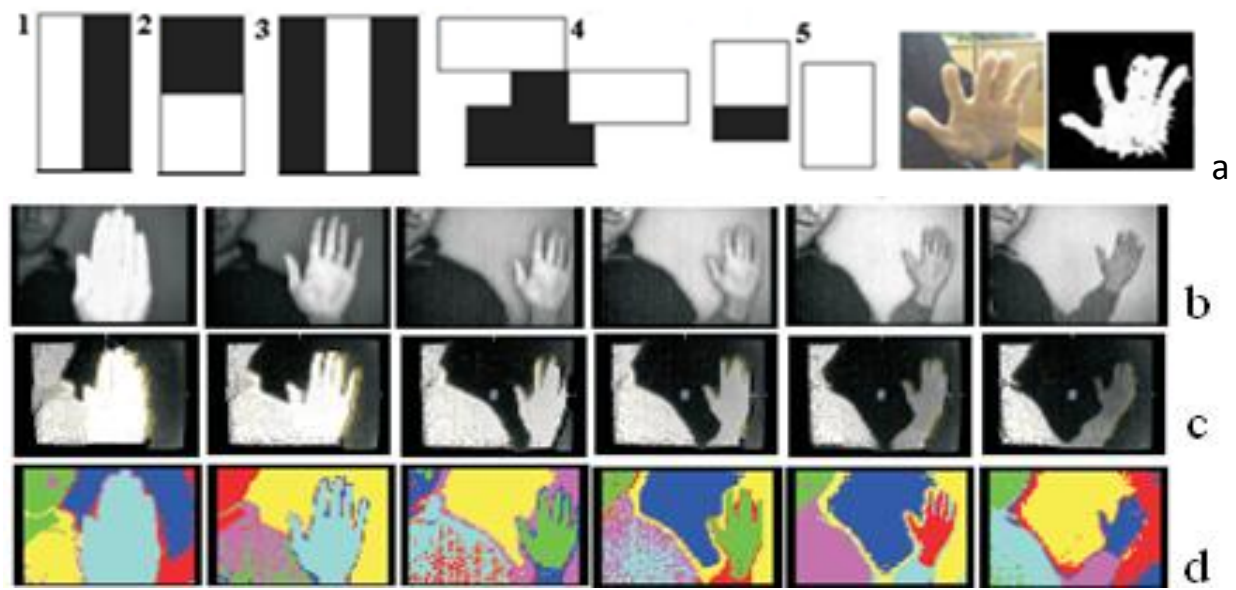

b
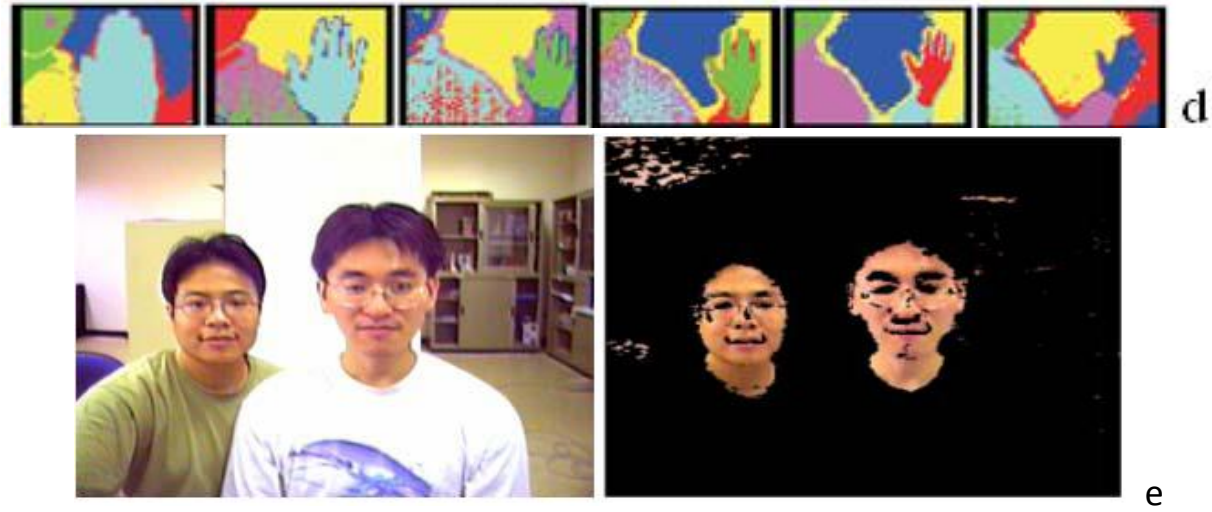

a) Modified Adaboost method for segmentation, the first three shapes is the original and the second two is the adopted new shapes [27]. b) Segmentation of a sequence of movement gesture, b) Intensity images, c) Range images, d) Segmented images [30]. e) Used two quadratic polynomials to approximate the upper and lower boundaries of the skin distribution plane [14].

Fig 6: Another set of results of modeling and segmentation of some discussed methods.

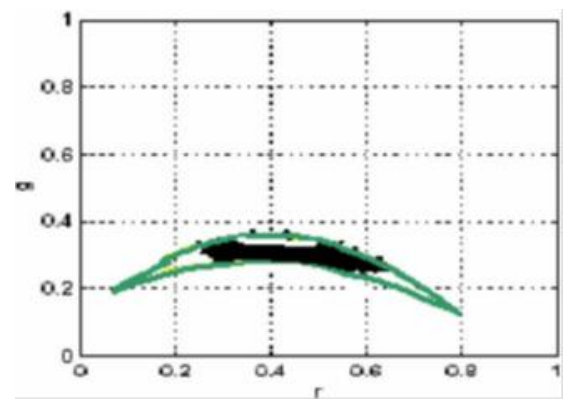

Fig 7: skin-color pixels distribution in r-g color space [14].

\section{CONCLUSION AND DISCUSSION}

A review of common techniques used for segmentation process is presented. Different application areas required segmentation process such as computer vision and objects recognition. A unified algorithm for segmenting colored images is not available since it depends on the required application area, however different segmentation techniques are adequate and performed efficiently for some applications. The diversity of color spaces provides the ability to select the proper color space that can be utilized well under different environment conditions. Segmentation based skin color is a simple and easy way to classify the image into skin and nonskin pixels, but on the other hand its sensitivity to illumination changes and the interference with background skin color imposed some hypothesis to work well. GM and GMM are statistical methods used recently for modeling skin color distribution in different applications. GMM classifier is discussed in this literature since it provides an acceptable performance result with minimum set of training data, and requires low storage system as well. In general, techniques based probabilistic modeling required higher computational cost and time since the pdf is calculated for each pixel in the entire image which will increase with the increasing of image size. Segmentation performance improved when GMM combined with histogram based Bayes methods [23][12], besides the use of chrominance color model only to achieve 
illumination invariant results [51][62][12][23]. Empirically [61] discovered that $\mathrm{YCbCr}$ has significant performance with GMM rather than RGB and normalized r-g color space. The combination of two complexion color models and background model using GMM introduce good results in an intricate background, but the result not perfect with the existence of high light and shadows [10]. GM, Bayes classifier with histogram and Neural network (NN) are evaluated in [21] and discovered that $\mathrm{NN}$ required less memory than Bayes classifier. Range information acquired from ToF camera also introduces accurate and efficient output segmentation results. Finally for any skin color based segmentation method the careful selection of color space and the model applied to build the distribution for the skin color image are the most important coefficients for efficient and accurate segmentation.

Table 4: Application of different segmentation methods over various color spaces.

\begin{tabular}{|l|l|l|}
\hline Ref\# & Color model used & Segmentation method used \\
\hline$[29]$ & Normalized RGB & Thresholding \\
\hline$[25]$ & YCbCr color space & Thresholding \\
\hline$[38]$ & Normalized YUV & Thresholding \\
\hline$[63]$ & HS model & $\begin{array}{l}\text { Discrete and Continuum } \\
\text { topological derivatives }\end{array}$ \\
\hline$[36]$ & - & $\begin{array}{l}\text { Feed forward back } \\
\text { propagation neural network, } \\
\text { and active contour for } \\
\text { tracking }\end{array}$ \\
\hline$[16]$ & - & Greedy algorithm and HMM \\
\hline$[39]$ & RGB & $\begin{array}{l}\text { Generic model and vector } \\
\text { machine SVM active } \\
\text { learning }\end{array}$ \\
\hline$[27]$ & HSV & $\begin{array}{l}\text { Modified Adaboost method } \\
\text { and optical flow and color } \\
\text { cues for tracking }\end{array}$ \\
\hline$[37]$ & $\begin{array}{l}\text { HVC in Munsell } \\
\text { color space }\end{array}$ & $\begin{array}{l}\text { Acquired skin color image } \\
\text { and intensity image and } \\
\text { multiplied them }\end{array}$ \\
\hline$[30]$ & $\begin{array}{l}\text { Range information } \\
\text { and intensity } \\
\text { images }\end{array}$ & $\begin{array}{l}\text { Combined K-means and EM } \\
\text { approaches }\end{array}$ \\
\hline$[14]$ & $\begin{array}{l}\text { Normalized RGB, } \\
\text { the r-g color model }\end{array}$ & $\begin{array}{l}\text { two quadratic polynomials } \\
\text { rule }\end{array}$ \\
\hline
\end{tabular}

\section{ACKNOWLEDGMENTS}

Our thanks to the experts who have contributed towards development of the template.

\section{REFERENCES}

[1] Robert M. Haralick, Linda G. Shapiro "Computer and Robot Vision”, Addison-Wesley, Co., Vol. 2, 1993.

[2] P.Daniel Ratna Raju, G.Neelima, K.Prasada Rao, "Image segmentation-MR Images Segmentation with A Modified Gaussian Mixture Model", International Journal of Computer Science and Information Technologies (IJCSIT), Vol. 2 (6) , pp. 2573-2578, 2011.

[3] Mantas Paulinas, Andrius Ušinskas, "A Survey of Genetic Algorithms Applications for Image
Enhancement And Segmentation", Information Technology And Control, Vol. 36(3), 2007. Available: http://itc.ktu.lt/itc363/paulinas363.pdf

[4] Siddhartha Bhattacharyya,"A Brief Survey of Color Image Preprocessing and Segmentation Techniques", Journal of Pattern Recognition Research,Vol. 6(1), pp. 120-129, 2011. Available: http://www.jprr.org/index.php/jprr/article/viewFile/191/9 6

[5] Qiang Wu, Fatima Merchant, Kenneth R. Castleman, "Microscope Image Processing", Academic Press, pages 9, April 2008

[6] Wikipedia website.

[7] Luigi Lamberti, Francesco Camastra, "Real-Time Hand Gesture Recognition Using a Color Glove", the 16th international conference on Image analysis and processing: Part I (ICIAP'11), Springer-Verlag Berlin Heidelberg, pp. 365-373, 2011.

[8] Francesca Gasparini, Raimondo Schettini, "Skin Segmentation Using Multiple Thresholding", In Internet imaging VII, IS and T/SPIE, pp. 60610F-1-60610F-8. SPIE 2006. Available: http://www.ivl.disco.unimib.it/papers2003/EI06EI109\%20Skin-paper.pdf

[9] Yu-Hsiang Wang, “Tutorial: Image Segmentation”, Graduate Institute of Communication Engineering National Taiwan University, Taipei, Taiwan. Available: http://disp.ee.ntu.edu.tw/meeting/\%E6\%98\%B1\%E7\%B F\%94/Segmentation\%20tutorial.pdf

[10] Shuying Zhao, Wenjun Tan, Shiguang Wen, and Yuanyuan Liu, "An Improved Algorithm of Hand Gesture Recognition under Intricate Background", the First International Conference on Intelligent Robotics and Applications (ICIRA 2008),: Part I. Springer-Verlag Berlin Heidelberg, pp. 786-794, 2008. Doi:10.1007/9783-540-88513-9_85

[11] Sudeep Sarkar, Barbara Loeding, Ruiduo Yang, Sunita Nayak, Ayush Parashar, "Segmentation-robust Representations, Matching, and Modeling for Sign Language", IEEE Computer Society Conference on Computer Vision and Pattern Recognition Workshops (CVPRW), pp. 13-19, June 2011. Doi: $\underline{\text { 10.1109/CVPRW.2011.5981695 }}$

[12] Zhan Gao, “Appearance-based Hand Gesture Detection”, available:

http://www.cs.auckland.ac.nz/courses/compsci705s1c/ex ams/SeminarReports/HandGesture_zgao014.pdf

[13] Sergio 'Alvarez, David F. Llorca, Gerard Lacey, Stefan Ameling, "Spatial Hand Segmentation Using Skin Color And Background Subtraction”, Trinity College Dublin's Computer Science Technical Report, Dublin, November 2010, Available: http://www.scss.tcd.ie/publications/techreports/reports.10/TCD-CS-2010-35.pdf

[14] Cheng-Chin Chiang, Wen-Kai Tai, Mau-Tsuen Yang, Yi-Ting Huang, Chi-Jaung Huang, "A novel method for detecting lips, eyes and faces in real time", Elsevier Real-Time Imaging, Vol. 9, pp. 277-287, 2003. Doi: $\underline{10.1016 / j . r t i .2003 .08 .003}$. 
[15] Mokhtar M. Hasan, and Pramod K. Mishra, "Hand Gesture Modeling and Recognition using Geometric Features: A Review", Canadian Journal on Image Processing and Computer Vision Vol. 3(1), March 2012. Available:

http://www.ampublisher.com/Mar\%202012/IPCV-1203015-Hand-Gesture-Modeling-Recognition-GeometricFeatures-Review.pdf

[16] Ruiduo Yang, Sudeep Sarkar, "Gesture Recognition using Hidden Markov Models from Fragmented Observations", IEEE Conference on Computer Vision and Pattern Recognition (CVPR'06), Vol. 1, 2006. Doi: 10.1109/CVPR.2006.126

[17] Michael J. Jones, James M. Rehg, "Statistical Color Models with Application to Skin Detection", IEEE Conference on Computer Vision and Pattern Recognition, Vol. 1, June 1999. Doi; $\underline{\text { 10.1109/CVPR.1999.786951 }}$

[18] L. Lucchese , S. K. Mitra, "Color Image Segmentation: A State-of-the-Art Survey", Indian national science academy, Vol. 67(2), pp.207-221, 2001. Doi: $\underline{10.1 .1 .84 .4896}$

[19] K S Deshmukh, , "Color image segmentation: a review", SPIE Second International Conference on Digital Image Processing, Vol. 7546, pp. 754624-754624-6, 2010. Singapore .Doi: $\underline{10.1117 / 12.856011}$

[20] Władysław Skarbek, Andreas Koschan, "Color Image Segmentation: A Survey", Berlin, Germany, October 1994. Available: http://imaging.utk.edu/ koschan/paper/coseg.pdf

[21] Son Lam Phung, Abdesselam Bouzerdoum, Douglas Chai, "Skin Segmentation Using Color Pixel Classification: Analysis And Comparison", IEEE Transactions on Pattern Analysis and Machine Intelligence, pp. 148-154, Vol. 27( 1), January 2005.

[22] Jean-Christophe Terrillon, Mahdad N. Shirazi, Hideo Fukamachi, Shigeru Akamatsu, "Comparative Performance of Different Skin Chrominance Models and Chrominance Spaces for the Automatic Detection of Human Faces in Color Images", IEEE the Fourth International Conference on Automatic Face and Gesture Recognition 2000 (FG '00), 2000.

[23] M. Van den Bergh, E. Koller-Meier, F. Bosch', L. Van Gool, "Haarlet-based Hand Gesture Recognition for 3D Interaction", Workshop on Applications of Computer Vision (WACV), pp. 1-8, 2009, doi: 10.1109/WACV.2009.5403103

[24] N. A. Ibraheem, M. M. Hasan, R. Z. Khan, P. K. Mishra, "Understanding Color Model: A Review", ARPN Journal of Science and Technology, vol. 2(4): 265-275, May 2012.

[25] E. Stergiopoulou, N. Papamarkos, "Hand Gesture Recognition Using A Neural Network Shape Fitting Technique", Elsevier, Engineering Applications of Artificial Intelligence, vol. 22 pp. 1141-1158, 2009 .doi:10.1016/j.engappai.2009.03.008

[26] Paola Campadelli, Francesco Cusmai, Raffaella Lanzarotti, "A Color-Based Method For Face Detection”, available:

http://homes.dsi.unimi.it/ lanzarot/Articoli/IST2003.pdf

[27] Yikai Fang, Kongqiao Wang, Jian Cheng, Hanqing Lu, "A Real-Time Hand Gesture Recognition Method", IEEE, ICME, pp. 995-998, 2007. Available: http://www.nlpr.labs.gov.cn/2007papers/gjhy/gh45.pdf

[28] Xiaojin Zhu Jie Yang Alex Waibel, "Segmenting Hands of Arbitrary Color", IEEE the Fourth IEEE International Conference on Automatic Face and Gesture Recognition (FG '000), pp. 446 - 453, 2000. Doi: 10.1109/AFGR.2000.840673

[29] WÃlodzimierz Kasprzak, Artur Wilkowski, and Karol Czapnik, "Hand Gesture Recognition in Image Sequences Using Active Contours and HMMs", Image Processing \& Communications Challenges, Academy Publishing House EXIT, Warszawa 2009, pp. 248-255.

[30] S. E. Ghobadi, O. E. Loepprich, K. Hartmann, and O. Loffeld, "Hand Segmentation Using 2D/3D Images", conference of Image and Vision Computing New Zealand 2007, pp. 64-69, New Zealand, December 2007.

[31] Vaishali S. Kulkarni, S.D.Lokhande, “Appearance Based Recognition of American Sign Language Using Gesture Segmentation", International Journal on Computer Science and Engineering (IJCSE), pp. 560-565, Vol. 2(3), 2010.

[32] Thiago R. Trigo and Sergio Roberto M. Pellegrino, "An Analysis of Features for Hand-Gesture Classification", 17th International Conference on Systems, Signals and Image Processing (IWSSIP 2010), pp. 412- 415, 2010

[33] Yepeng Guan, Mingen Zheng, "Real-time 3D pointing gesture recognition for natural HCI", IEEE the seventh World Congress on Intelligent Control and Automation, pp. 2433-2436, June 2008. Doi: 10.1109/WCICA.2008.4593304

[34] G. Awad, T. Coogan, J. Hann, A. Sutherland, "RealTime Hand Gesture Segmentation, Tracking and Recognition", School of Computing, Dublin City University, Dublin 9, Ireland.

[35] Gorodnichy, Dimitry; Yogeswaran, A., "Detection and Tracking of Pianist Hands and Fingers", IEEE The 3rd Canadian Conference on Computer and Robot Vision, pp. 63-63, June 2006. doi; 10.1109/CRV.2006.26

[36] M. K. Viblis, K. J. Kyriakopoulos, "Gesture Recognition: The Gesture Segmentation Problem", Journal of Intelligent and Robotic System, vol. 28, pp. 151-158, June 2000. Doi: 10.1023/A:1008101200733

[37] Shinji Tsuruoka, Akio Kinoshita, Tetsushi Wakabayashi, Yasuji Miyake, Muneaki Ishida, "Extraction of Hand Region and Specification of Finger Tips from Color Image", IEEE International Conference on Virtual Systems and MultiMedia (VSMM '97), pp. 206 - 211, Augest 1997, doi: 10.1109/VSMM.1997.622348

[38] R. G. O' Hagan, A. Zelinsky, S. Rougeaux, "Visual Gesture Interfaces For Virtual Environments", Elsevier, interacting with computers, Vol. 14, pp. 231-250, 2002, doi: 10.1016/S0953-5438(01)00050-9

[39] Junwei Han, George M. Award, Alistair Sutherland, and Hai $\mathrm{Wu}$, "Automatic Skin Segmentation for Gesture 
Recognition Combining Region and Support Vector Machine Active Learning”, IEEE 7th International Conference on Automatic Face and Gesture Recognition (FGR'06), pp. 237 - 242, April 2006. Doi: 10.1109/FGR.2006.27

[40] Mahmoud Elmezain, Ayoub Al-Hamadi, J"org Appenrodt, and Bernd Michaelis, "A Hidden Markov Model-Based Isolated and Meaningful Hand Gesture Recognition”, World Academy of Science, Engineering and Technology, Vol. 41, 2008.

[41] M.-H. Yang and N. Ahuja, "Gaussian Mixture Model for Human Skin Color and Its Applications in Image and Video Databases," SPIE Storage and Retrieval for Image and Video Databases, vol. 3656, pp. 458-466, Jan. 1998. Doi: $\underline{10.1117 / 12.333865}$

[42] Rahman Farnoosh, Behnam Zarpak, "Image Segmentation Using Gaussian Mixture Model", International Journal of Engineering Science (IUST), pp. 29-32, Vol. 19(1-2), 2008.

[43] Krishna Kant Singh ,Akansha Singh, “A Study Of Image Segmentation Algorithms For Different Types Of Images", International Journal of Computer Science Issues IJCSI, Vol. 7(5),September 2010.

[44] Chapter 4 Segmentation. Available: http://www.bioss.ac.uk/staff/chris/ch4.pdf

[45] Fritz Albregtsen, "Region and Edge Based Segmentation", 2010.

[46] Vladimir Vezhnevets, Vassili Sazonov, Alla Andreeva, "A Survey on Pixel-Based Skin Color Detection Techniques", IN PROC. GraphiCon-2003. Available: http://graphics.cs.msu.ru/en/publications/text/gc2003vsa. pdf

[47] Son Lam Phung, Chai, D., Bouzerdoum, A., “A universal and robust human skin color model using neural networks", International Joint Conference on Neural Networks, (IJCNN '01), Washington, DC, July 2001.Doi: $\underline{10.1109 / \mathrm{IJCNN} .2001 .938827}$

[48] Jander Moreira, Luciano Da Fontoura Costa, "Neuralbased color image segmentation and classification using self-organizing maps", 1996. Available: http://sibgrapi.sid.inpe.br/col/dpi.inpe.br/ambro/1998/04. 17.15.45/doc/a19.pdf

[49] Constantino Carlos Reyes-Aldasoro, Anal Aura Aldeco, "Image Segmentation and Compression Using Neural Networks", available http://www.cisst.org/ cista/446_2004/papers/SegAndCo mpression.pdf

[50] Bir Bhanu, Sungkee Lee, John Ming, "Adaptive Image Segmentation Using a Genetic Algorithm", IEEE transaction on systems, man, and cybernetics, Vol. 25(12), pp. 1543- 1567.December 1995.

[51] Demir Gokalp, "Learning Skin Pixels in Color Images Using Gaussian Mixture", available http://www.cs.bilkent.edu.tr/ guvenir/courses/cs550/Wor kshop/Demir_Gokalp.pdf

[52] Bertrand Scherrer, "Gaussian Mixture Model Classifiers", 2007.
[53] Mohand Saïd Allili, "A short tutorial on Gaussian Mixture Models", CRV 2010. available http://www.computerrobotvision.org/2010/tutorial_day/ GMM_said_crv10_tutorial.pdf

[54] Shanthi Devara, Ron Kneusel, "ExpectationMaximization”, CSCI 5454 Lecture 19 April 11, 2011.

[55] Nikhil R. Pal, Sankar K. Pal, "A Review on Image Segmentation Techniques", Elsevier Pattern Recognition Vol. 26(9), pp. 1277-1294, may 1993.doi: 10.1016/0031-3203(93)90135-J.

[56] Noor Adnan Ibraheem., Rafiqul Zaman Khan, "Vision Based Gesture Recognition Using Neural Networks Approaches: A Review", International Journal of Human Computer Interaction (IJHCI) Malaysia, Vol. 3(1), 2012.

[57] Hassana Grema Kaganami, Zou Beiji, "Region-Based Segmentation versus Edge Detection", IEEE Fifth International Conference on Intelligent Information Hiding and Multimedia Signal Processing, pp. 12171221, 2009. DOI: $10.1109 / \mathrm{IIH}-\mathrm{MSP} .2009 .13$

[58] http://homepages.inf.ed.ac.uk/rbf/CVonline/LOCAL_CO PIES/RAMANI1/node19.html

[59] Bryan S. Morse, "Lecture 18: Segmentation (Region Based)", 2002.

[60] Image Segmentation, Lecture 16.

[61] Hebert Luchetti Ribeiro, Adilson Gonzaga, "Hand Image Segmentation in Video Sequence by GMM: a comparative analysis" IEEE on Computer Graphics and Image Processing (SIBGRAPI'06), 2006

[62] Stephen J. Mckenna, Shaogang gong, Yogesh Raja, "Modeling Facial Color and Identity with Gaussian Mixtures", Elsevier, April 1998.

[63] M.Krishnaveni, V.Radha, "Topological Derivative Based Image Segmentation For Sign Language Recognition System Using Isotropic Filter", International Journal of Computer Science and Information Security(IJCSIS), Vol. 6( 3), 2009.

[64] Nguyen N.T., Bui T.D., "Automated Posture Segmentation In Continuous Finger Spelling Recognition", IEEE 3rd International Conference on Human-Centric Computing, HumanCom 2010, August 2010. Cebu. Doi: 10.1109/HUMANCOM.2010.5563311

[65] Qiulei Dong, Yihong Wu and Zhanyi Hu, "Gesture Segmentation from a Video Sequence Using Greedy Similarity Measure", IEEE 18th International Conference on Pattern Recognition (ICPR 2006). pp. 331 - 334, September 2006. doi: 10.1109/ICPR.2006.608

[66] Yining Deng and B. S. Manjunath, "Unsupervised Segmentation of Color-Texture Regions in Images and Video", IEEE Transactions on Pattern Analysis and Machine Intelligence, Vol. 23(8), August 2001. Doi: $\underline{10.1109 / 34.946985}$

[67] http://homepages.inf.ed.ac.uk/rbf/HIPR2/log.htm

[68] Rafiqul Zaman khan, Noor Adnan Ibraheem, "Survey on Gesture Recognition for Hand Image Postures", Canadian Center of Computer and Information Science, Vol. 5(3), pp. 110-121, May 2012. doi: $10.5539 /$ cis.v5n3p110 